PALEO

Revue d'archéologie préhistorique

$26 \mid 2015$

Varia

\title{
Du nouveau aux Combarelles I (Les Eyzies-de- Tayac, Dordogne, France)
}

Something new in Combarelles I (Les Eyzies-de-Tayac, Dordogne, France

Elena Man-Estier, Emeline Deneuve, Patrick Paillet, Lucie Loiseau et Catherine Cretin

\section{(2) OpenEdition}

\section{Journals}

Édition électronique

URL : http://journals.openedition.org/paleo/2987

DOI : $10.4000 /$ paleo.2987

ISSN : 2101-0420

Éditeur

SAMRA

\section{Édition imprimée}

Date de publication : 1 décembre 2015

Pagination : 201-214

ISSN : $1145-3370$

\section{Référence électronique}

Elena Man-Estier, Emeline Deneuve, Patrick Paillet, Lucie Loiseau et Catherine Cretin, « Du nouveau aux Combarelles I (Les Eyzies-de-Tayac, Dordogne, France) », PALEO [En ligne], 26 | 2015, mis en ligne le 26 avril 2016, consulté le 07 juillet 2020. URL : http://journals.openedition.org/paleo/2987 ; DOI : https://doi.org/10.4000/paleo.2987

\section{(c) (i) (9)}

PALEO est mis à disposition selon les termes de la licence Creative Commons Attribution - Pas d'Utilisation Commerciale - Pas de Modification 4.0 International. 


\title{
Du nouveau aux Combarelles I (Les Eyzies-de-Tayac, Dordogne, France)
}

\author{
Elena MAN-ESTIER ${ }^{(a)}$, Emeline DENEUVE ${ }^{(\mathrm{b})}$, Patrick PAILLET ${ }^{(\mathrm{c})}$, \\ Lucie LOISEAU(d) ${ }^{(\mathrm{d})}$ Catherine CRETIN(e)
}

\begin{abstract}
Résumé : Située au cœur de la vallée de la Vézère, la grotte des Combarelles I (Les Eyzies-de-Tayac, Dordogne, France) est connue pour les gravures souvent enchevêtrées qui se déploient de part et d'autre d'un long et unique couloir. Les études conduites jusqu'à présent ont permis de souligner la diversité des thèmes figurés et l'organisation du dispositif pariétal. La principale technique d'expression est la gravure. Des représentations dessinées ou peintes en noir et en rouge sont également connues. Cependant, elles ont toujours été perçues comme des éléments secondaires et anecdotiques du décor. Mettant à profit des moyens d'analyses innovants, notamment par le biais des traitements colorimétriques de l'image, nous avons débuté une nouvelle étude du corpus peint de la cavité. Nous présentons ici les premiers résultats de nos observations, l'inventaire établi en 2014 ainsi qu'un exemple concret d'application : un cheval réalisé selon une technique mixte (gravure/peinture) dans la partie profonde de la cavité.
\end{abstract}

Mots-clés : Art préhistorique, Les Combarelles, Magdalénien, dessin, peinture, techniques d'expression, traitements numériques de la couleur.

Abstract: Something new in Combarelles I (Les Eyzies-de-Tayac, Dordogne, France). Situated in the valley of the Vézère, the cave of Combarelles I (Les Eyzies-de-Tayac, Dordogne, France) is known for its tangled engravings which are organized on both sides of the walls of a long corridor. Studies conducted until now emphasized the diversity of figurative themes expressed throughout a relative organization of the dispositive. Engravings are the main expression technique. The presence of black and red drawings or paintings is also known. Curiously, it has never been seen as an important element of the ornementation.Taking advantage of new technological medium of analysis, including colorimetric processing of the pictures, we began a new study on the painted corpus of the cavity. We are here presenting the first results of the inventory realized in 2014 and a concrete application example: a horse made of a mixed technique (drawing/engraving), in the deepest part of the cavity.

Key-words: Prehistoric art, Les Combarelles, Magdalenian, drawing, painting, expression techniques, digital processing.

(a) Sous-direction de l'archéologie, Ministère de la culture et de la communication et UMR 5199 - PACEA elena.man-estier@culture.gouv.fr

(b) Institut national de Recherches en archéologie préventive, Ministère de la culture et de la communication emeline.deneuve@inrap.fr

(c) Muséum national d'Histoire naturelle - Musée de l'Homme, UMR 7194 - HNHP - paillet@mnhn.fr

(d) Université de Bordeaux I, UMR 5199 - PACEA - loiseau.lucie40@gmail.com

(e) Centre national de Préhistoire, Sous-direction de l'Archéologie, Ministère de la culture et de la communication et UMR 5199 PACEA. - catherine.cretin@culture.gouv.fr 


\section{Les Combarelles I, découverte et contexte}

La grotte des Combarelles I s'ouvre en rive gauche de la Beune, affluent de la Vézère, au fond d'un petit vallon. Elle est située à trois kilomètres en amont du bourg des Eyziesde-Tayac et à quelques centaines de mètres de la grotte de Font-de-Gaume (fig. 1). L'entrée, constituée par un large porche, donne accès à deux galeries ornées indépendantes, Combarelles I et Combarelles II (Aujoulat 1984 ; Barrière 1984 ; Roussot 1976). Au fond de Combarelles I, une troisième zone ornée (Combarelles III) est découverte en 2005 par Marc Delluc (Cleyet-Merle, Feruglio, Delluc sous presse). La grotte des Combarelles I est creusée dans le même calcaire du Coniacien que plusieurs autres cavités de la région. Mais la morphologie de la grotte des Combarelles I est un peu différente. Elle est assez longue $(249 \mathrm{~m})$ pour le seul réseau fossile, et se présente comme un unique couloir sinueux « en zigzag » (Barrière 1997) (fig. 2). La galerie est de largeur modeste (entre 0,60 et $1,50 \mathrm{~m}$ ). Au Paléolithique sa hauteur variait de 0,5 à 1,5 m, localement $2 \mathrm{~m}$. En 1911, dix ans après la découverte, le sol est abaissé de plus de 0,50 m pour faciliter l'accès aux visiteurs. La roche support, de couleur beige clair, est recouverte assez régulièrement d'une pellicule de calcite dont l'aspect varie d'un blanc opaque à un gris translucide en fonction de l'humidité et des conditions climatiques extérieures. Dans la dernière partie de la galerie, les parois sont recouvertes d'argile de décalcification, parfois entamée par la gravure et partiellement recouverte de calcite.

La grotte des Combarelles I fait partie des tout premiers sites ornés reconnus dans la vallée de la Vézère au début du $X X^{e}$ siècle. II s'agit même de la première grotte « inventée » conjointement par Louis Capitan, Henri Breuil et Denis Peyrony, le futur « trust des grottes ornées » (Hurel 2011). Elle convainc la communauté scientifique de l'existence et de l'ancienneté de l'art paléolithique. La grotte est découverte le 8 septembre 1901 et signalée à l'Académie des Sciences le 18 septembre (fig. 3) (Capitan et Breuil 1901 ; Cleyet-Merle 1990). Les premières publications (Capitan et Breuil 1902a, 1902b et 1902c) font état d'une centaine de représentations. Quelques gravures remarquables sont signalées, comme les 14 mammouths qui constituent un argument fort en faveur de l'âge paléolithique du site. L'étude menée par la suite, dont H. Breuil est le maître d'œuvre, complète le corpus (Capitan, Breuil, Peyrony 1924). À partir de 1978, une nouvelle étude est conduite par Claude Barrière. Ses relevés sont publiés dans plusieurs volumes des Travaux de l'Institut d'Art préhistorique de Toulouse (Barrière 1980, 1981, 1983a,b, 1985 et 1987). Une version commentée et enrichie d'une synthèse est présentée dans un numéro hors série de la revue Paléo (Barrière 1997). À partir des années 1980, Monique et Claude Archambeau procèdent à de nouvelles lectures et publient des entités graphiques inédites (Archambeau M. 1984 ; Archambeau M. et C. 1986, 1991).

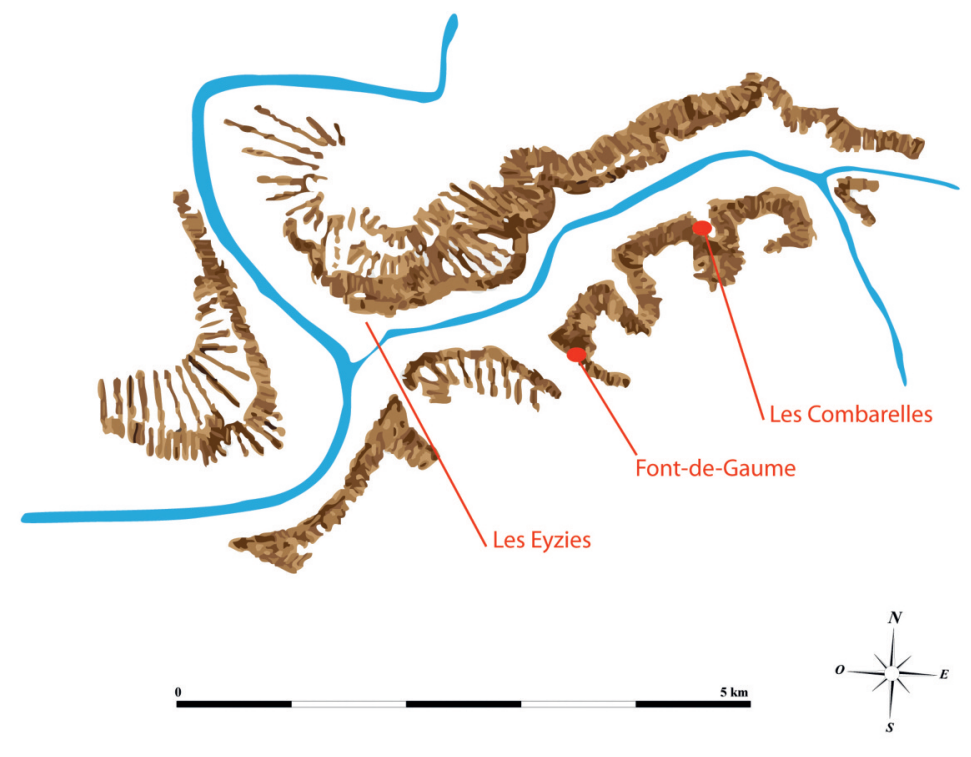

Figure 1 - Localisation de la grotte des Combarelles. DAO E. Deneuve, d'après Barrière, 1997.

Figure 1 - Localisation of the cave of Combarelles. Drawing E. Deneuve after Barrière 1997. 


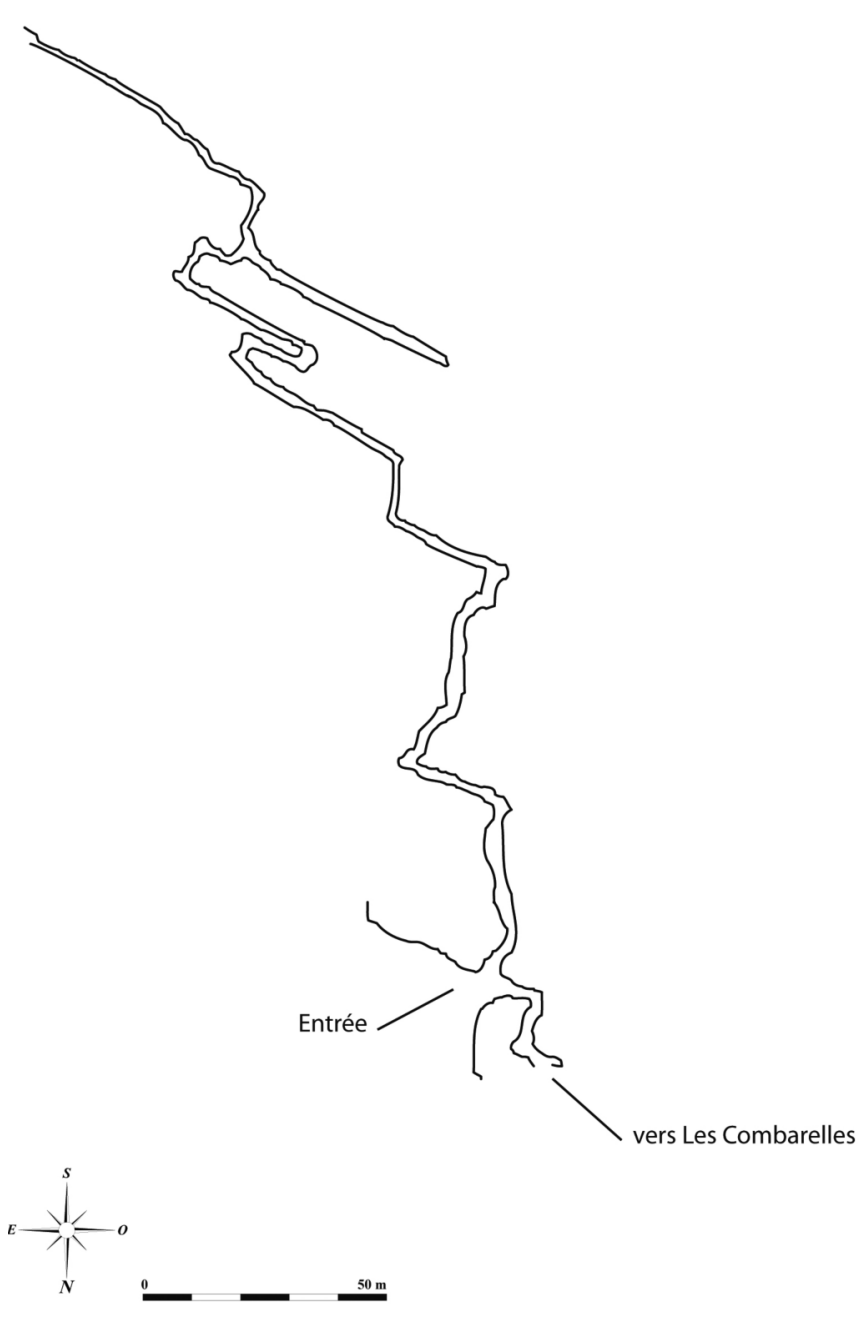

Figure 2 - Plan de la grotte des Combarelles I. DAO E. Deneuve, d'après Barrière 1997.

Figure 2 - Map of Les Combarelles I. Drawing E. Deneuve after Barrière 1997.

Le contexte archéologique du site est mal connu. Dès 1892, le porche et les galeries font l'objet de fouilles par Émile Rivière (Rivière 1894). Le rare matériel recueilli confirme la fréquentation au Paléolithique supérieur. En 1973, une fouille de sauvetage est menée par JeanPhilippe Rigaud (1984). II met en évidence les lambeaux de deux niveaux d'occupation, datés respectivement entre 13698 et 12784 ans cal BP pour le niveau supérieur, et 17174 et 15939 ans cal BP pour le niveau inférieur ${ }^{1}$.

Le style des représentations et la présence de certains marqueurs thématiques (signes tectiformes, figures

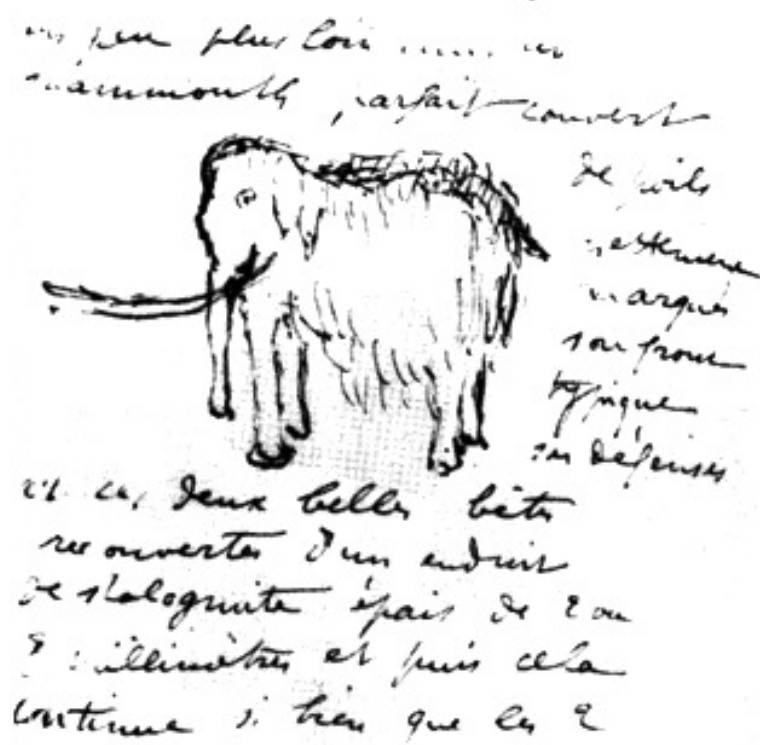

Figure 3 - Lettre du Dr Capitan relatant la découverte des Combarelles I, In : Cleyet-Merle 1990.

Figure 3 - Letter written by Dr Capitan explaining the discovery of Les Combarelles I, In: Cleyet-Merle 1990.

féminines schématiques) conduit l'ensemble des auteurs à proposer une période de réalisation entre le Magdalénien moyen et le Magdalénien supérieur, c'est-à-dire aux alentours de 15000 ans BP non cal.

\section{Le corpus orné des Combarelles I : gravures et peintures}

La principale originalité de l'art des Combarelles I est l'enchevêtrement des gravures (fig. 4). Le décor est présent dès les premières dizaines de mètres du réseau mais il se concentre surtout dans les 100 derniers mètres de la galerie. Cet ensemble est caractérisé par une forte densité et une continuité iconographiques rarement interrompues par des accidents de la paroi ou par les coudes qui jalonnent le parcours.

L'inventaire global publié par H. Breuil (Capitan, Breuil, Peyrony 1924) mentionne près de 300 représentations. En 1902 L. Capitan et H. Breuil signalent que « sur quelques figures, les traits gravés sont rehaussés d'une bande étroite de peinture noire qui parfois les remplace » (Breuil et Capitan 1902). En 1924, c'est une trentaine de " dessins » qui est répertoriée. Parmi ces derniers, 16 représentations figuratives sont individualisées et parfois déterminées (chevaux, bouquetin et signes notamment). Claude Barrière double ce corpus général tout en récusant

(1) Niveau supérieur $11380+/-210$ ans BP (LY3201) et niveau inférieur $13680+/-210$ ans BP (LY3202). Calibrations réalisées grâce au logiciel OxCal et à la courbe IntCal13 (Reimer et al. 2013). 


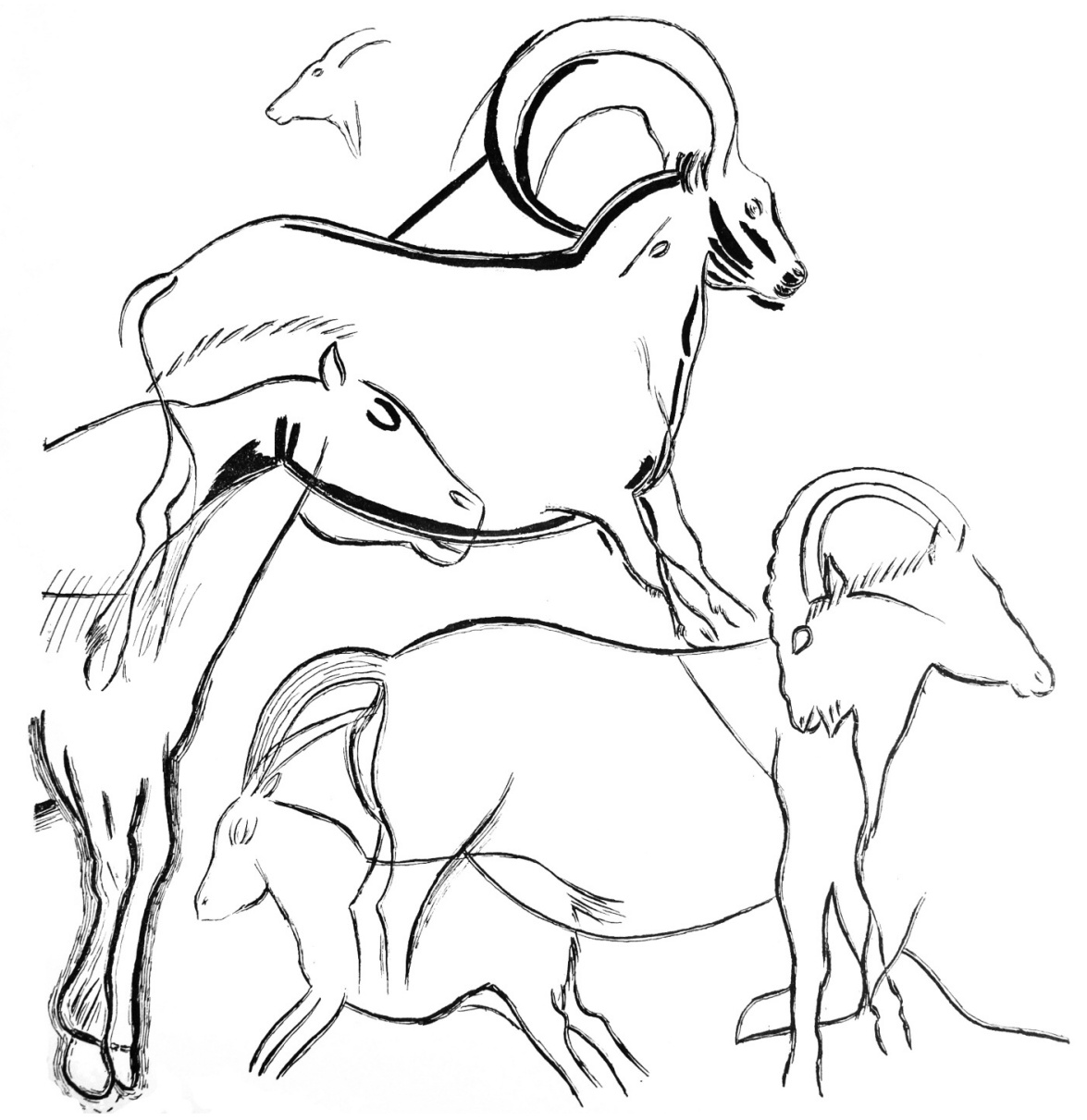

Figure 4 - Panneau gravé de la grotte des Combarelles I, relevé H. Breuil.

Figure 4 - Engraving panel of Les Combarelles I, tracing $\mathrm{H}$. Breuil. certaines figures anciennement publiées. Son inventaire (1997) comprend 625 motifs répartis en 529 gravures et 96 dessins. Les « figures », très majoritairement animalières (Barrière 1997 - p. 474), sont au nombre de 460. L'inventaire des dessins figuratifs (16) est équivalent à celui de H. Breuil (fig. 5).

Dans le cadre d'une nouvelle opération archéologique, nous avons eu l'opportunité de procéder à une relecture des dessins $^{2}$ et de compléter l'inventaire de C. Barrière. Une relecture détaillée des parois a permis d'individualiser une trentaine de motifs peints supplémentaires, portant ce corpus à 121 unités graphiques. II s'agit en très grande majorité d'entités non déterminables et très effacées : taches, points ou encore segments de tracés discontinus (fig. 6).

Si l'on considère l'ensemble des représentations des Combarelles I, le corpus est largement dominé par le Cheval. Claude Barrière en recense 141 , soit près d'un tiers du corpus figuratif et plus de $20 \%$ de l'ensemble des entités graphiques. Au sein du corpus peint, les chevaux occupent également une place dominante (9 sur 16 figurations).

Les humains constituent le second thème $(n=55)$. Les formes de représentation sont variées : humain complet, segments (sexes, mains), têtes bestialisées, figures féminines schématiques. Monique et Claude Archambeau (1991) classent ces humains en quatre groupes (figurations humaines expressives, énigmatiques, géométriques et indéterminées).

Le reste du corpus figuratif est assez classique. Les inventaires de C. Barrière (1997) font état de 42 bovinés, dont 38 bisons, de 24 mammouths et 21 cervidés. Enfin, on peut noter la présence d'espèces plus rares dans le bestiaire magdalénien, comme l'ours $(n=9)$ (ManEstier 2009), le lion $(n=3)$, les canidés $(n=3)$, la hyène $(n=1)$ et un Mustélidé. Enfin, le corpus des signes abstraits s'établit à 69 entités graphiques.

(2) Nous employons indifféremment les termes de « dessin » ou "peinture ». La méthode d'application du pigment semble davantage correspondre à de la peinture au trait qu'à du dessin, cependant l'utilisation de ce terme dans la littérature nous conduit à l'employer également. 


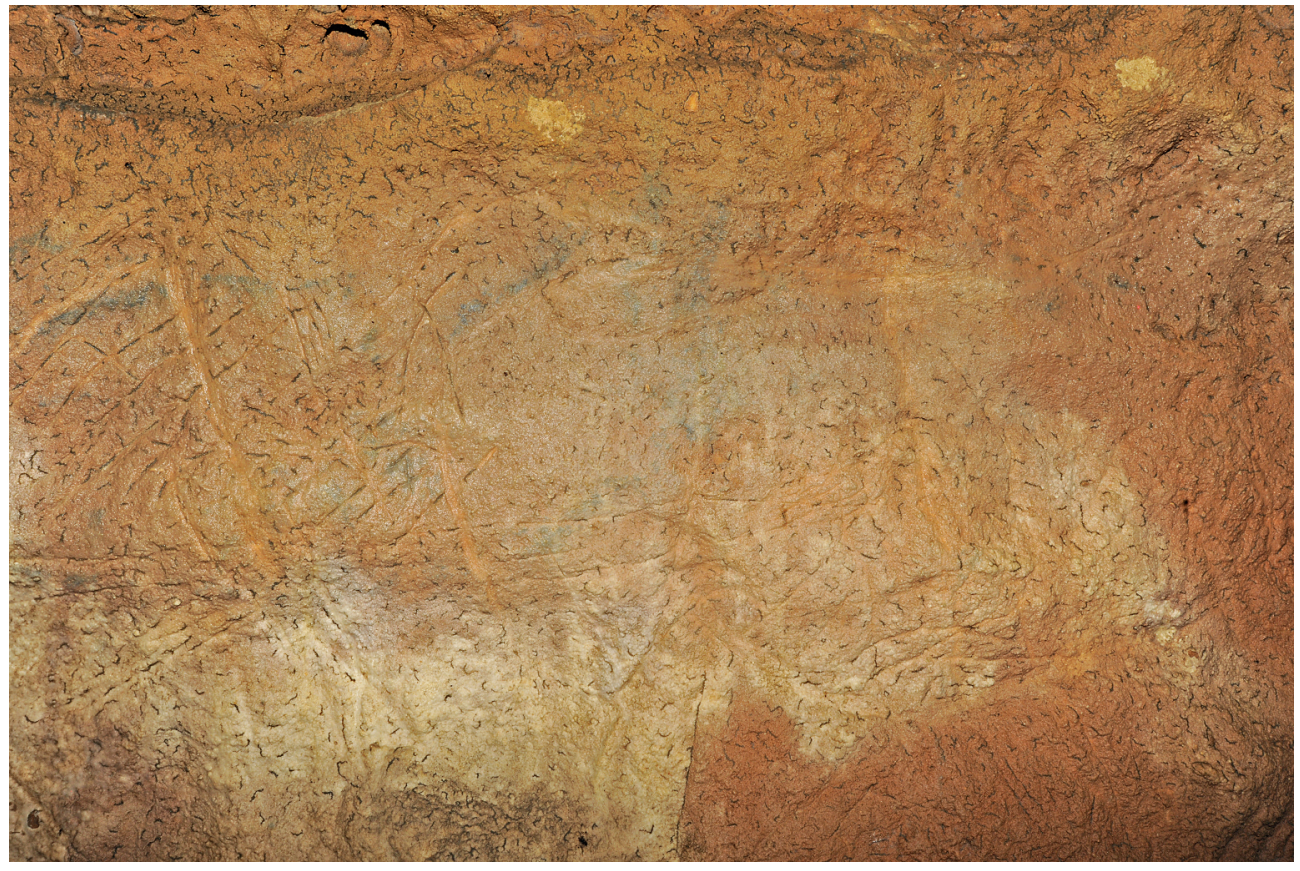

Figure 5 - Dessins noirs de la grotte des Combarelles I, cliché Ph. Jugie (@ CMN).

Figure 5 - Black drawings in Les Combarelles I, picture Ph. Jugie (@ CMN).

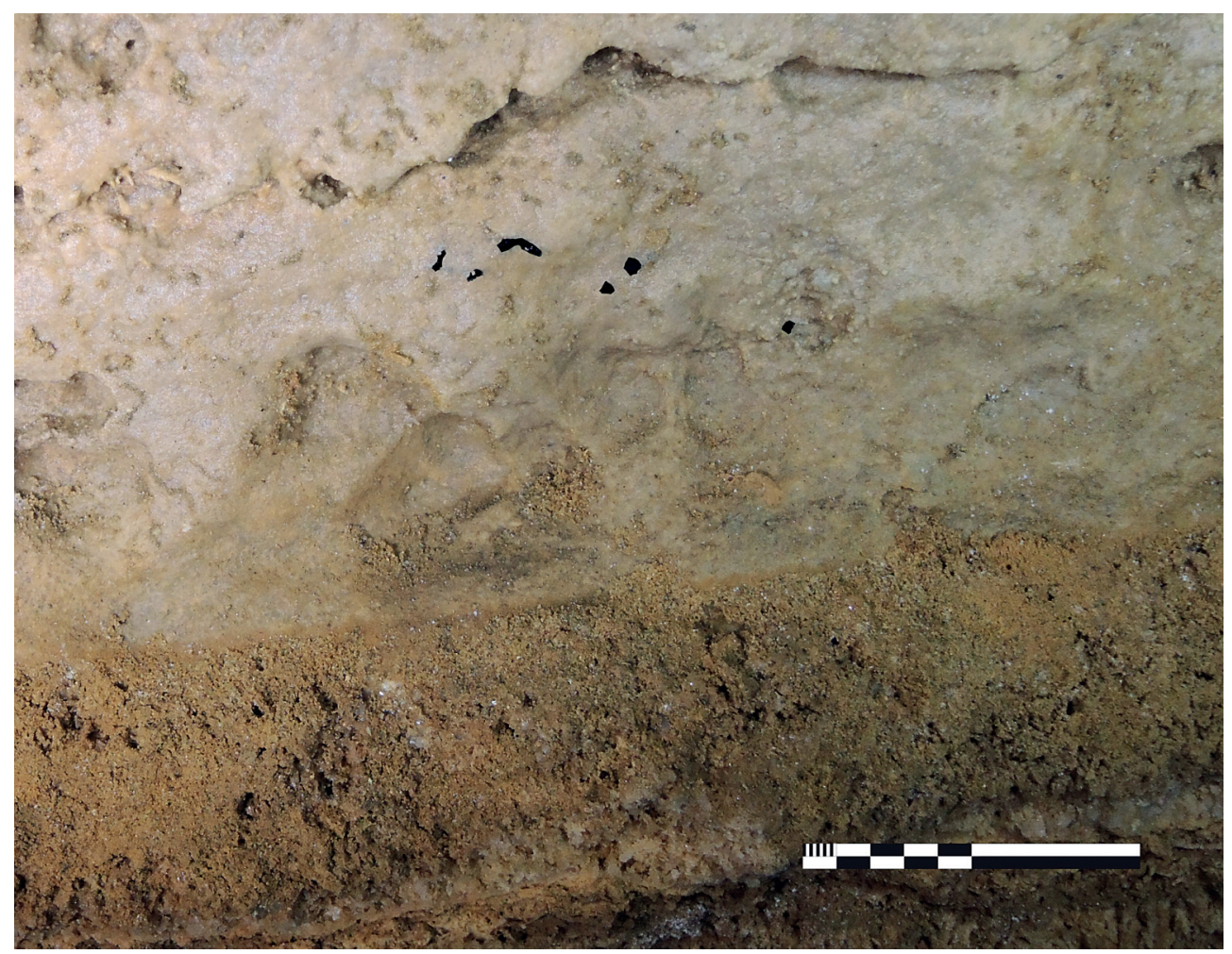

Figure 6 - Un motif noir inédit de la grotte des Combarelles I. Traitement D-Stretch @ amélioré, cliché P. Paillet, DAO E. Man-Estier.

Figure 6 - New black pattern in Les Combarelles I. Improved D-Strech treatment. Picture P. Paillet, treatment E. Man-Estier. 


\section{Une nouvelle problématique et de nouveaux outils}

La grotte a subi de nombreuses altérations naturelles qui ont endommagé les parois de manière plus ou moins importante. Des recouvrements de calcite blanchâtre ont eu lieu en période humide et se produisent encore aujourd'hui. Peu visibles sous la couche de calcite, les dessins semblent avoir perdu beaucoup de leur intérêt pour les anciens chercheurs : « 5 dessins, c'est peu de chose » (Barrière 1997 - p. 514).

Ainsi, mis à part quelques tracés encore identifiables, ils ont été perçus comme anecdotiques, limités et sans interférence avec le corpus gravé. Claude Barrière choisit de les publier séparément des gravures, en réalisant deux séries de relevés différents.

Mais pour prendre toute la mesure du dispositif orné, il nous semble indispensable d'associer les dessins et les gravures. C'est dans cette optique que nous avons proposé une nouvelle étude ${ }^{3}$ dont les premiers résultats sont présentés ici.

Notre analyse est basée sur un inventaire tiré des prospections systématiques des parois et d'un double enregistrement, photographique et graphique, des représentations identifiées. En parallèle, un traitement d'image numérique est appliqué aux représentations pour améliorer la lisibilité des tracés. Nous avons utilisé le plugin D-Stretch (C) du logiciel ImageJ (c) (Harmann 2011). Son utilisation est désormais répandue dans la communauté scientifique notamment pour l'étude de l'art rupestre. Plusieurs auteurs en ont largement développé l'intérêt avant nous (Brady et al. 2012 ; Defrasne 2014 ; Hollmann et Crause 2011 ; Le Quellec et al. 2103 ; Le Quellec, Duquesnoy, Defrasne 2015). II est vrai que cet outil, libre et régulièrement mis à jour, offre une interface d'utilisation plutôt simple pour des résultats souvent spectaculaires. Nous avons systématisé l'utilisation de ce logiciel en l'insérant dans notre analyse du corpus des Combarelles I, après la première étape de prospection et d'inventaire en paroi. Une seconde étape de post-production sur ordinateur permet d'améliorer encore le résultat obtenu par D-Strech (C) et de le superposer à la photo originale. Le résultat des traitements numériques est spectaculaire tant il permet de rendre visible ce qui est presque invisible aujourd'hui. II ne s'agit toutefois que d'un travail informatique, qui n'est en aucun cas destiné à remplacer l'observation in situ et le relevé.

\section{Un corpus actualisé}

En 2014, nous avons identifié 121 entités graphiques colorées. Nous nous sommes concentrés sur les parties médianes et profondes de la grotte (secteurs $\mathrm{VI}$ à $\mathrm{X}$ de C. Barrière), caractérisées dès 1901 comme les principales zones ornées. Sur ce total, 31 entités sont inédites. Trois autres sont évoquées par C. Barrière (1997) mais non figurées dans les publications.

Nous avons considéré comme « unité graphique » tout motif inscrit dans un espace délimitable par des lacunes dans le décor ou des accidents naturels. Certaines représentations semblent homogènes d'un point de vue technique ou thématique, mais s'inscrivent dans un espace important, jusqu'à plusieurs mètres. Elles sont enregistrées en tant qu'unité graphique globale, subdivisée le cas échéant pour en faciliter la description. Une attention particulière a été portée au vocabulaire utilisé pour décrire les techniques d'expression en usage. Nos définitions s'appuient sur celles proposées dans l'ouvrage rédigé par le Groupe de Réflexion sur l'Art Pariétal Paléolithique (Collectif, 1993) et dans le Dictionnaire de la Préhistoire (Vialou dir. 2004). Nous avons ainsi distingué les taches, les points ou les traits, des aplats colorés. Les différences entre dessins et peintures relèvent des propres modalités de préparation et d'application du pigment qui nous sont inconnues à ce stade de l'étude.

La majeure partie des éléments identifiés est noire $(n=110)$. Le pigment a été parfois projeté sur la paroi $(n=24)$. Il s'agit le plus souvent de peinture au trait $(n=51)$. Plusieurs marques circonscrites $(n=45)$ évoquent un tamponnage (fig. 7). La chronologie de réalisation des tracés est souvent difficile à établir. Toutefois, sur une dizaine de représentations qui combinent peinture et gravure, nous avons pu réaliser quelques observations qui contredisent les hypothèses antérieures. En effet, alors que $\mathrm{H}$. Breuil ne détaille pas les séquences chronologiques, C. Barrière indique que " toutes les traces colorées sont antérieures aux gravures » (Barrière 1983 - p. 69). En fait, il ne s'agit en aucun cas d'une constante. Au contraire, plusieurs représentations présentent une chronologie complexe qui permet de proposer la contemporanéité des gravures et des peintures.

Les unités graphiques de couleur rouge sont peu nombreuses $(n=11)$ et majoritairement limitées à des taches ou des ponctuations. Toutefois, plusieurs ensembles plus construits, que l'on peut qualifier de signes, sont dessinés ou peints en rouge. C'est le cas des deux plus grands signes du dispositif pariétal, un rectangle cloisonné juxtaposé à la lionne VIIIG104 et un motif élaboré considéré comme un "pseudo-tectiforme » en fin de dispositif (XDD42). Nous avons observé globalement davantage d'éléments rouges sur la paroi gauche que sur celle de droite, avec une plus forte concentration dans le secteur VII, entre 176 et 181 mètres de l'entrée.

(3) «Les dessins noirs et rouges de Combarelles I », Relevé d'art rupestre, 2014 et 2015, E. Man-Estier, dir. Ce programme s'inscrit dans le cadre du Projet Collectif de Recherche "Archéologie des sites ornés de Dordogne : cadre conceptuel, potentiel et réalités ", 2014-2016, coordonné par C. Cretin. 

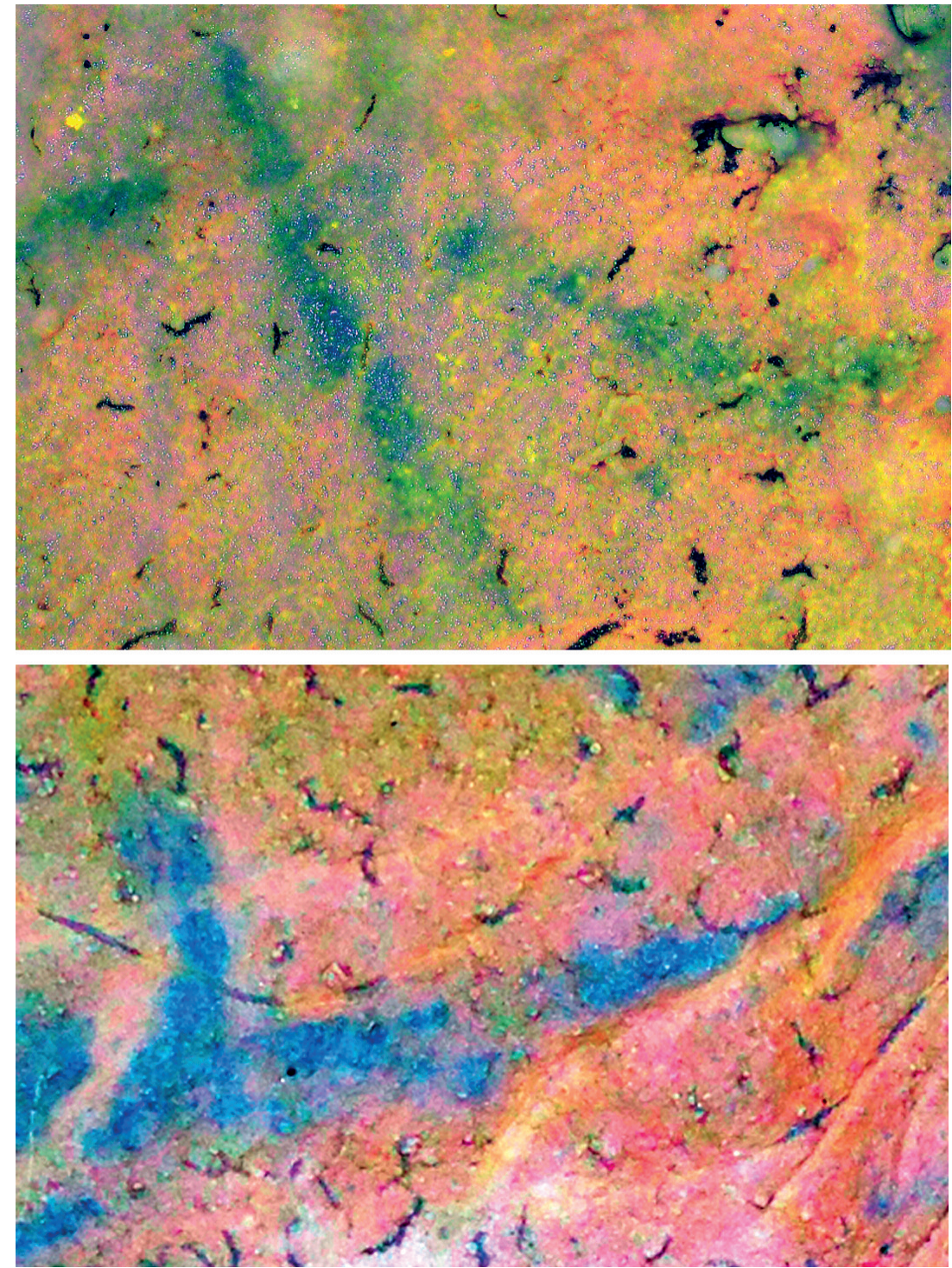

Figure 7 - Différentes techniques de dessin mises en évidence par le traitement numérique. En haut, trait ponctué, en bas tracé au pinceau. Traitement $D$-Stretch $(\mathcal{C}$, cliché P. Paillet.

Figure 7 - Several drawing techniques enlighted with digital treatment. Up, line made of ponctuation, down, painted line. D-Strech treatment. Picture P. Paillet.
Parmi les 121 dessins recensés, nous avons relevé 32 représentations figuratives. Ce total comprend les peintures qui forment par elles-mêmes un motif figuratif (essentiellement animal) ou qui s'inscrivent au sein d'une représentation figurative gravée, en présentant un lien thématique avec elle (fig. 8). Les chevaux sont les plus fréquents (14 représentations sûres et 10 autres probables). Les autres thèmes sont les rennes $(n=4)$, les félins $(n=2)$, le mammouth $(n=1)$ et le bouquetin $(n=1)$ (fig.9).

Trois motifs abstraits présentent une construction complexe. Le premier, dessiné en noir, est associé à un bison gravé (fig. 10). Les deux autres sont de couleur rouge, sans lien avec des gravures (fig. 11). II existe également une main négative placée à l'entrée de la partie terminale de la cavité. Elle est partielle et située dans une « niche » de la voûte (fig.12).
L'essentiel du corpus est constitué de tracés indéterminés $(n=87)$. II s'agit de traits isolés, de taches ou encore de nappes de couleur. Trois tracés constituent de probables segments d'animaux (tête et rachis).

Les liens thématiques entre gravures et dessins constituent un important sujet de réflexion. En ce qui concerne le cheval, nous avons noté 22 cas de superpositions. II s'agit le plus souvent de motifs partiels, placés à des endroits précis du corps de l'animal : lignes de contours ou points anatomiques comme l'épaule ou l'aine. En complément, 35 chevaux gravés sont associés à des marques colorées sans que l'on puisse mettre en évidence un lien anatomique. Enfin, deux chevaux semblent exclusivement dessinés (fig. 13).

En ce qui concerne les autres thèmes, un mammouth dessiné vient en parfait complément d'un proboscidien gravé. Six mammouths gravés sont associés à des marques colorées. Trois rennes présentent une association 


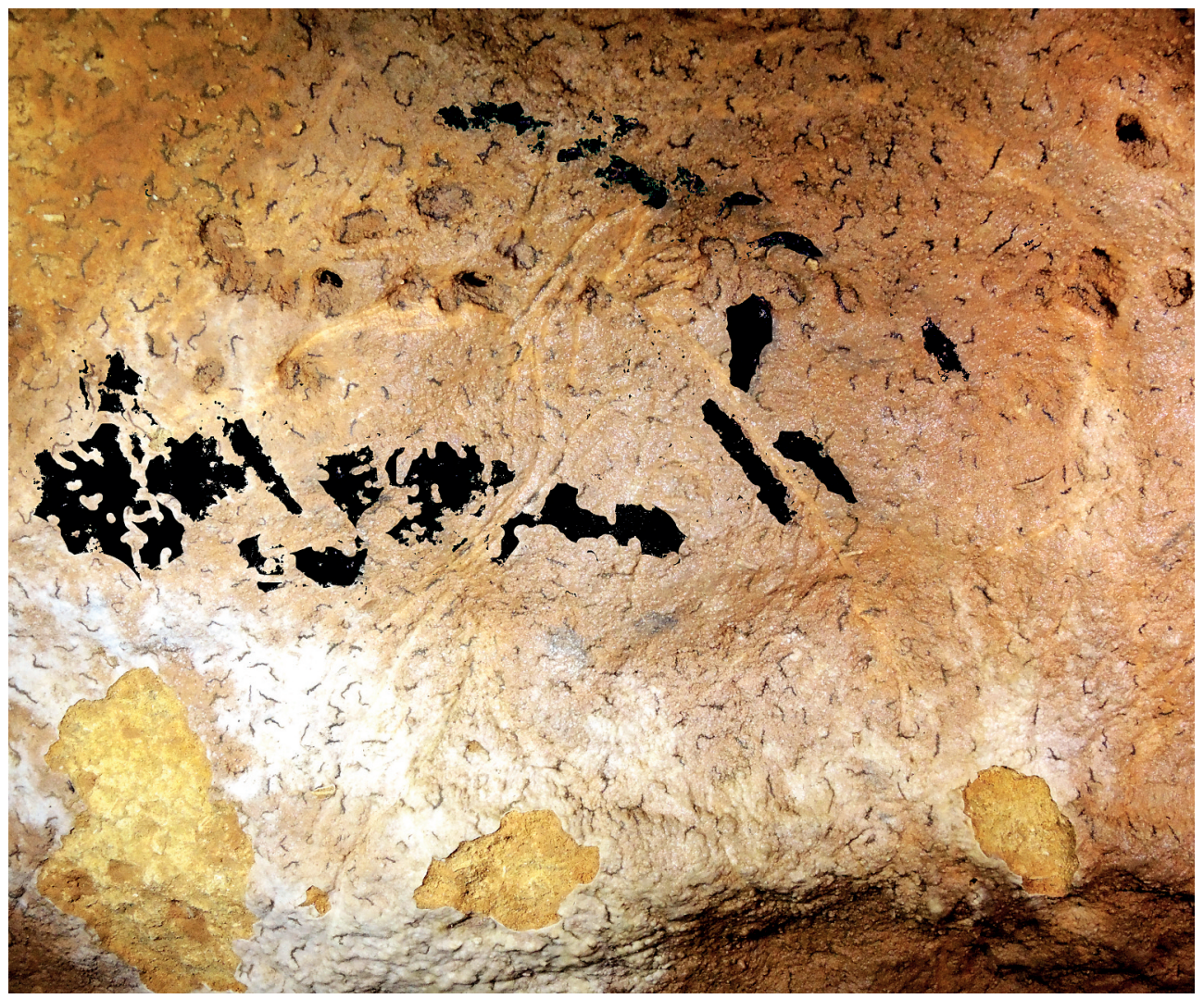

Figure 8 - Exemple de liaison thématique. La tête de cheval VID47 (inventaire C. Barrière) a été superposée au cheval dessiné Cb32. Traitement D-Stretch (C) amélioré, cliché P. Paillet, DAO E. Man-Estier.

Figure 8 - Example of thematic relation. Head of horse VID47 (C. Barriere) that has been superposed with drawn horse Cb32. Improved $D$-Strech treatment. Picture

P. Paillet, treatment E. Man-Estier.

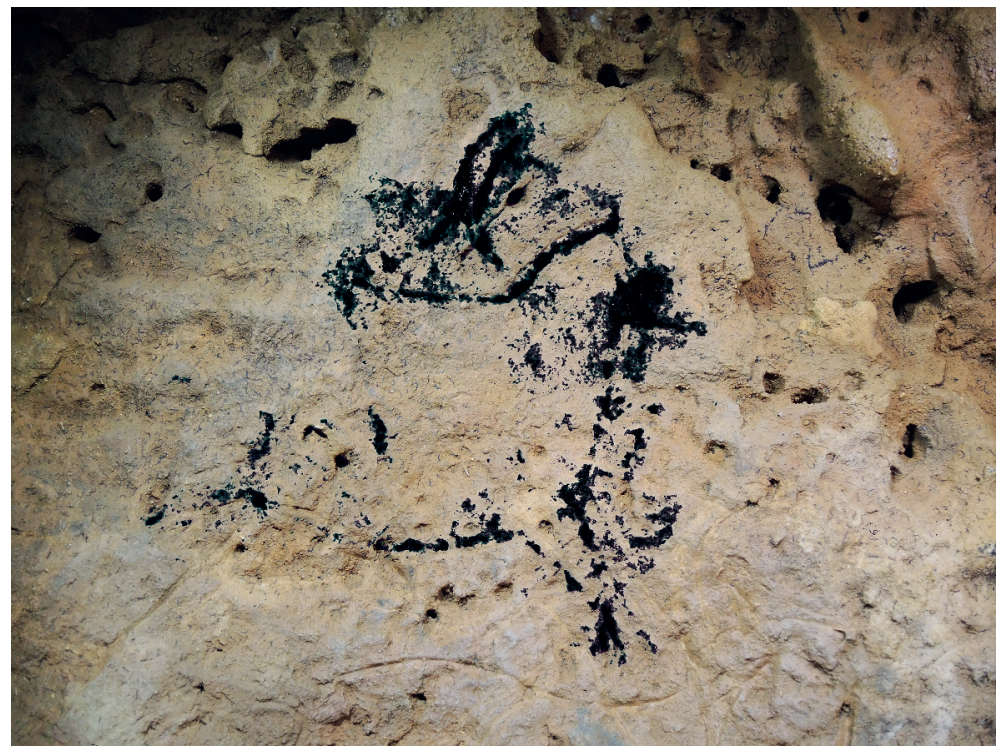

Figure 9 - Le bouquetin 67. Traitement D-Stretch (C) amélioré, cliché P. Paillet, DAO E. Man-Estier.

Figure 9 - Ibex 67. Improved D-Strech treatment. Picture P. Paillet, treatment E. Man-Estier.

de gravure et de couleur. Deux autres portent des vestiges de couleur dont on ne peut préciser s'il s'agit véritablement d'élément anatomique. Un renne est uniquement dessiné, sans lien avec les gravures alentours. Les deux lions gravés possèdent des taches noires : l'œil pour la "lionne », deux points sur le corps pour le lion qui la précède. Des marques colorées se superposent à trois bisons gravés, mais il n'existe aucun bison peint. Un bouquetin présente une association soignée entre gravure et peinture au trait (fig.4 et fig.9). Enfin, le dessin d'un animal non déterminable, sans lien avec une figure gravée, ainsi qu'un animal indéterminé gravé portant des vestiges de couleur complètent ces observations.

Des représentations humaines gravées et des marques colorées sont associées sur cinq panneaux de la grotte. 

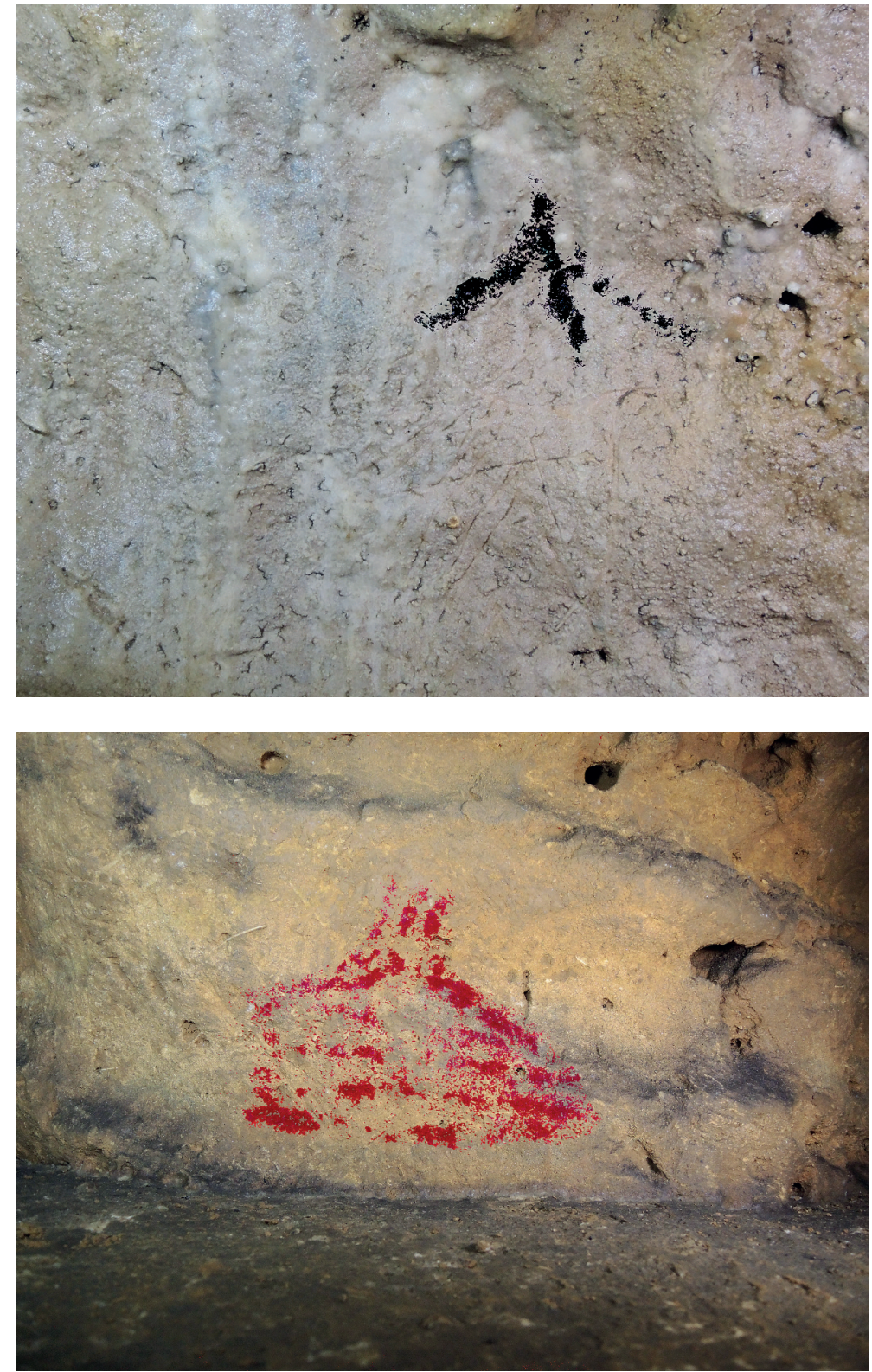

Figure 10 - Le signe 46. Traitement D-Stretch (C) amélioré, cliché P. Paillet, DAO E. Man-Estier.

Figure 10 - Sign 46. Improved D-Strech treatment Picture P. Paillet, treatment E. Man-Estier.
Notons également les points rouges sur les anthropomorphes de la " conque » entre les secteurs VI et VII.

L'étude de la localisation des entités graphiques dessinées permet de mettre en évidence une réelle continuité dans l'utilisation des pigments, qui ne sont pas réservés à une partie de la cavité. Le secteur $X$ est cependant, le plus dense (49 unités graphiques). Cela pourrait correspondre à un problème de conservation différentielle dans cette zone, qui est loin de l'entrée et fermée à la visite.

Les parois gauche et droite portent de la couleur dans une proportion à peu près équivalente. Nous avons recensé au total 62 entités graphiques sur la paroi de gauche et 59 sur la paroi de droite. Quelques éléments ont également été repérés au plafond. II s'agit essentiellement de taches, bien que quelques motifs semblent plus organisés.

\section{Un exemple de réalisation mixte : le cheval 89}

Le cheval 89 (XGD45 de la numérotation Barrière) est situé dans le fond de la grotte, sur la paroi gauche, dans la partie médiane du secteur $X$. La représentation mesure $45 \mathrm{~cm}$ de long et est placée à une cinquantaine de $\mathrm{cm}$ au-dessus du sol actuel. Elle s'appuyait originellement sur le sol archéologique.

L'animal est disposé en profil droit et orienté vers le fond de la grotte (fig. 14). II est entier et parfaitement détaillé. Sa position statique est soulignée par les pattes raides et le 


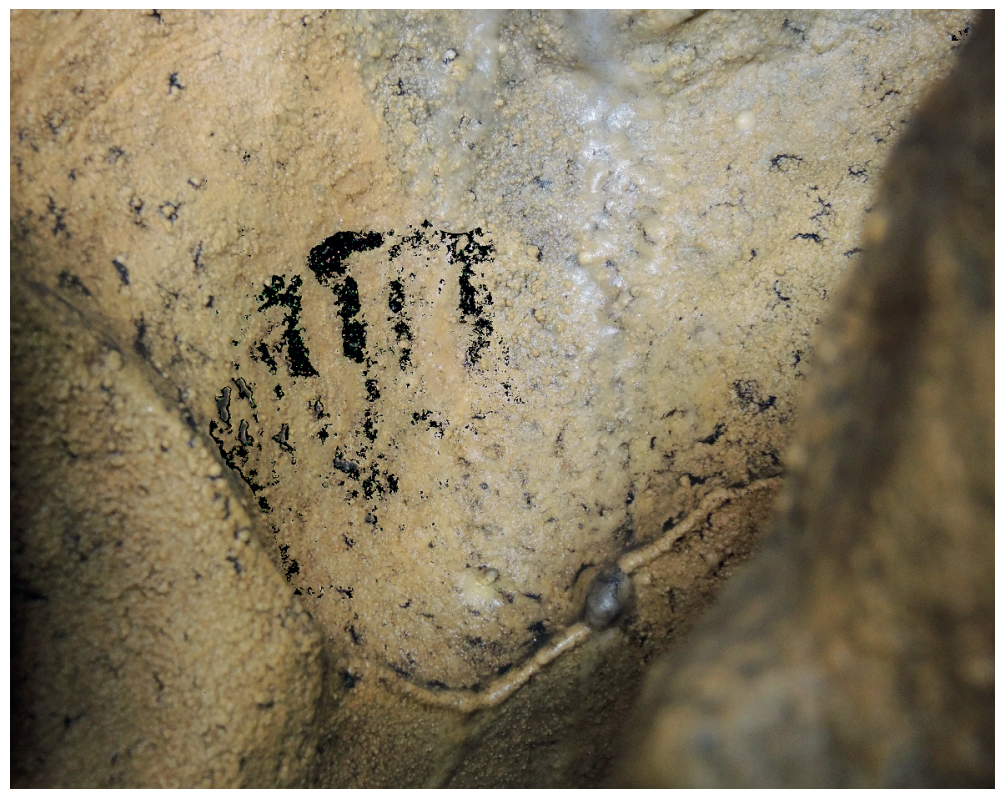

Figure 12 - La main négative 65.

Traitement D-Stretch (C) amélioré, cliché P. Paillet, DAO E. Man-Estier.

Figure 12 - Negative hand 65

Improved D-Strech treatment. Picture P. Paillet,

treatment E. Man-Estier

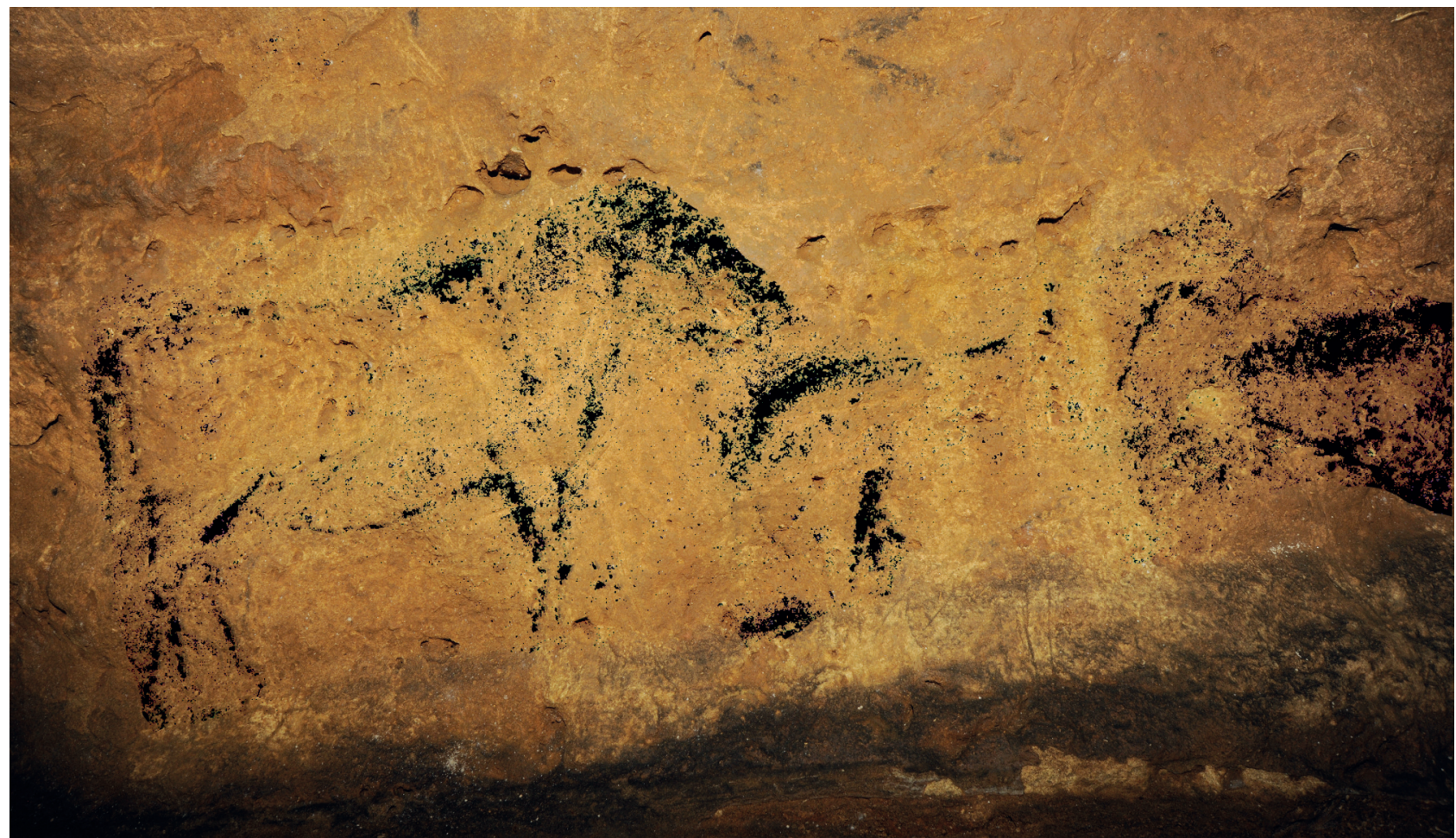

Figure 13 - Les chevaux 109 et 110. Traitement D-Stretch () amélioré, cliché P. Paillet, DAO E. Man-Estier.

Figure 13 - Horses 109 and 110. Improved D-Strech treatment. Picture P. Paillet, treatment E. Man-Estier.

port de tête droit. L'animal présente une morphologie trapue. La joue et le museau sont bien marqués. La tête est fine et complétée par le naseau, l'œil et l'oreille. La crinière est hérissée. L'épaule est marquée par un tracé épais, perpendiculaire au corps.

Le contour dorsal est arrondi, avec une ensellure légèrement marquée. Les fesses sont convexes et la cuisse s'insère avec un léger décrochement qui correspond parfaitement à l'anatomie de l'animal modèle. À l'avant comme à l'arrière, les deux membres sont présentés légèrement croisés. La queue, fine et pendante est également indiquée.

Cette représentation a été réalisée par une double technique de peinture au trait noir et de gravure. La mise en 


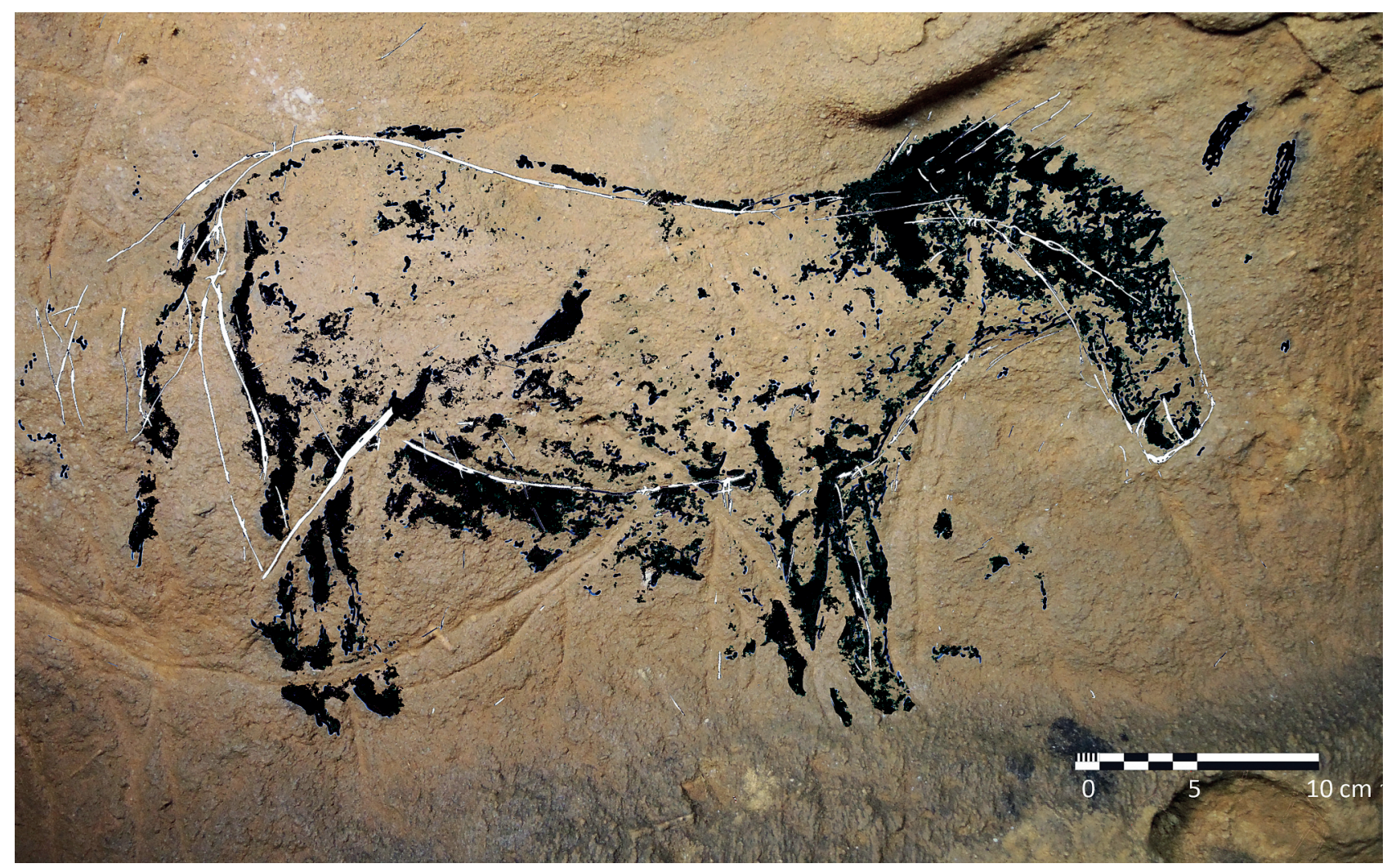

Figure 14 - Le cheval Cb89. Cliché P. Paillet, relevé et DAO E. Man-Estier.

Figure 14 - Horse 89. Picture P. Paillet, tracing E. Man-Estier.

place de techniques mixtes est connue dans l'art pariétal, même si elle n'est pas fréquente. II ne s'agit pas d'une peinture dont quelques détails auraient été rehaussés par gravure, ni d'une œuvre gravée présentant quelques taches de couleur (fig. 15). Au contraire, l'animal a été tracé par ces deux techniques différentes, qui se complètent ou se superposent. L'étude des superpositions permet de proposer une chronologie en trois temps. Nous avons noté une première phase de gravure fine, au niveau de l'encolure, de la crinière et de la ligne dorsale. C'est en quelque sorte le gabarit général de la représentation qui est positionné sur la paroi. Cette esquisse préalable est réalisée par gravure légère. Le motif est ensuite mis en couleur. Le dessin vient indiquer la tête, les contours des membres et du corps ainsi que la crinière. Certains détails anatomiques, comme l'oreille, sont alors indiqués. La succession des deux techniques est particulièrement notable le long de la ligne dorsale où l'on observe un écart (jusqu'à $2 \mathrm{~cm}$ ) entre les tracés gravé et peint (fig. 15). On note enfin une seconde phase de gravure, plus appuyée. L'artiste trace le contour de la tête en cernant scrupuleusement la couleur. II complète le tracé de la croupe et de la cuisse. Enfin, il précise le ventre, retraçant son contour dans la large bande ventrale réalisée auparavant. Le modelé noir de la tête et la marque de l'épaule ne trouvent pas pour le moment leur place dans cette chaîne opératoire graphique.

Un grand cheval, tourné en sens inverse, est gravé sur la paroi après la réalisation du cheval 89 . On remarque surtout son arrière-main, l'amorce des membres postérieurs et la ligne de ventre qui oblitèrent en grande partie le petit cheval précédent.

Du point de vue de la technique de dessin, nous avons noté que les traits noirs de contour sont d'épaisseur variable, de quelques millimètres à presque $2 \mathrm{~cm}$. La crinière, la tête et la partie inférieure du corps sont traitées en aplats colorés. La crinière est indiquée par une teinte noire très dense alors que les modelés du reste du corps sont plus diffus. Cela pourrait être dû à une conservation différentielle, mais il pourrait également s'agir de la mise en pratique de deux techniques légèrement différentes, comme une dilution plus ou moins importante du pigment. Par ailleurs, certains tracés noirs plus fins, comme l'oreille, pourraient avoir été exécutés au crayon ou à l'aide d'un pinceau fin.

Une poursuite des recherches et un recours à l'expérimentation permettront peut-être de préciser ces variations. 


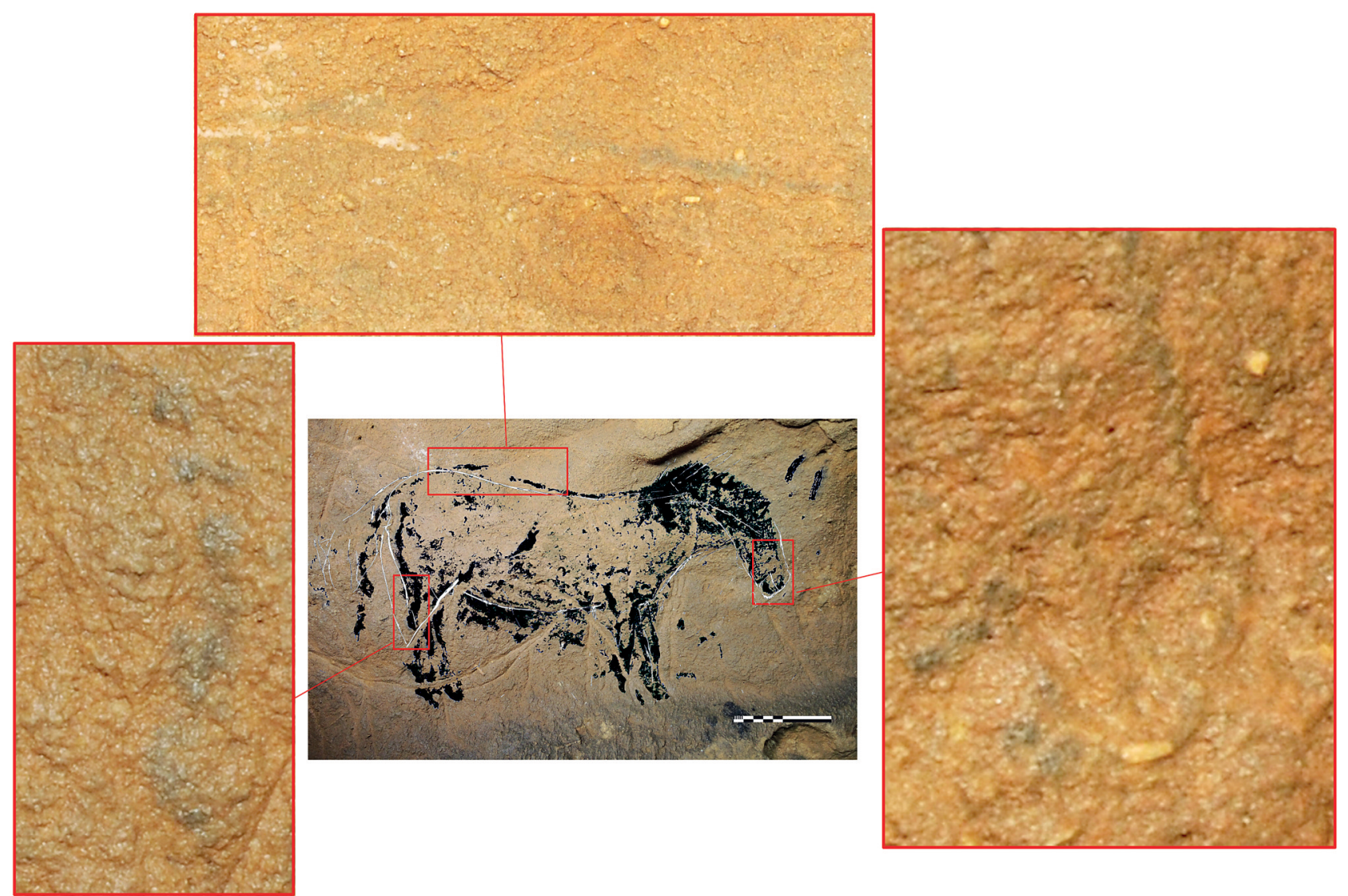

Figure 15 - Détails des tracés du cheval Cb89. Clichés Ph. Jugie (С CMN).

Figure 15 - Details of horse 89. Pictures Ph. Jugie (@ CMN).

\section{Conclusions provisoires}

Ce cheval, déjà bien connu, se montre aujourd'hui sous un jour nouveau. La mise en évidence du soin apporté aux détails anatomiques nous renseigne tout particulièrement sur l'importance des tracés peints aux yeux des artistes des Combarelles. De plus, l'existence d'une véritable technique mixte suggère qu'il s'agit d'un même ensemble ornemental, réalisé en une fois et formant un registre artistique et symbolique homogène dans l'esprit des préhistoriques. II nous faut alors repenser notre vision du décor de la grotte. C'est bien en noir (couleur de la peinture) et blanc (couleur de la gravure) que les artistes se sont exprimés aux Combarelles. Ils ont parfois ajouté du rouge pour rehausser encore leur réalisation.

Cette note s'appuyant sur un programme de recherches en cours a pour objectif de rappeler l'utilité et l'intérêt d'une relecture de site orné. Certaines technologies constituent en outre un précieux soutien aux analyses de l'art. Ce nouvel essor a débuté par la photographie numérique, s'est poursuivi par les techniques d'enregistrement tridimensionnelles, et par l'amélioration des rendus colorimétriques, qui permettent également de faciliter la lecture de ce qui ne se voit pas, ou plutôt de ce qui se voit si peu.

Pour autant, aucun ordinateur ne remplacera jamais l'acuité de l'œil, moins encore notre capacité de structuration des informations et d'analyse. Le temps gagné grâce à ces nouveaux outils est donc précieux, car en simplifiant les modalités de travail, il offre plus de temps au chercheur pour regarder la paroi et révéler les œuvres de la Préhistoire.

\section{Remerciements}

Les auteurs remercient Jean-Jacques Cleyet-Merle et Georges Levy ainsi que l'équipe des guides (Centre des Monuments nationaux) pour avoir autorisé et facilité l'accès à la cavité, Geneviève Pinçon (Centre National de Préhistoire) pour avoir rendu possible le stage de Master de Lucie Loiseau et John Harmann pour son autorisation d'utiliser le logiciel DStretch ( $)$. Cette opération est financée par le Ministère de la Culture et de la Communication, Sous-direction de l'Archéologie - DRAC Aquitaine. 


\section{Références bibliographiques}

ARCHAMBEAU M. 1984 - Les figurations humaines pariétales périgourdines : étude d'un cas, Les Combarelles, Thèse de doctorat de l'Université d'Aix en Provence, 2 vol., $235 \mathrm{p}$.

ARCHAMBEAU M. et C. 1986 - «Analyse typologique des figurations humaines de la grotte des Combarelles ", L'Anthropologie, 90, 4, Masson, Paris, pp. 805-806,1 fig.

ARCHAMBEAU M. et C. 1991 - « Les figurations humaines pariétales de la grotte des Combarelles », Gallia Préhistoire $\mathrm{n}^{\circ} 33$, pp. 53-81, 43 fig.

AUJOULAT N. 1984 - "Grotte des Combarelles II. Grotte à Mentoune. Grotte de Tounialou ", L'art des cavernes, Atlas des grottes ornées paléolithiques françaises, Imprimerie Nationale, Ministère de la Culture, Paris, Atlas archéologiques de la France, p. 114-118, 8 fig.

BARRIÈRE C.1980 - "La grotte des Combarelles : quelques gravures nouvelles ", Travaux de l'Institut d'Art Préhistorique, XXII, Toulouse, p. 137-148

BARRIÈRE C. 1981 - « La grotte des Combarelles. Relevés des gravures pariétales de la paroi ", Travaux de I'Institut d'Art Préhistorique, XXIII, Toulouse, p. 13-96.

BARRIÈRE C. 1983a - « Les peintures pariétales de Combarelles I, paroi gauche ", Travaux de l'Institut d'Art Préhistorique, XXV, Toulouse.

BARRIÈRE C. 1983b - "Combarelles I, les peintures pariétales de la galerie antérieure ", Travaux de l'Institut d'Art Préhistorique, XXV, Toulouse.

BARRIÈRE C. 1984 - «Grotte des Combarelles I. Grotte à Mentoune. Grotte de Tounialou. L'art des cavernes », Atlas des grottes ornées paléolithiques françaises, Imprimerie Nationale, Ministère de la Culture, Paris, Atlas archéologiques de la France, p. 109-113, 14 fig.

BARRIÈRE C. 1985 - « Les gravures pariétales de Combarelles I, la galerie profonde ", Travaux de l'Institut d'Art Préhistorique, XXVII.

BARRIÈRE C. 1987 - «Les dessins de la galerie profonde de Combarelles I », Travaux de l'Institut d'Art Préhistorique, XXIX.

BARRIÈRE C. 1992 - «Les Combarelles », La naissance de l'art en Europe, catalogue, Union Latine, Paris, p. 263265.

BARRIÈRE C. 1997 - L'art pariétal des grottes des Combarelles, Paléo hors-série n 1, 609 p.

BRADY L.M., GUNN R.G., MCDONALDS J., VETH P. 2012, "Digital enhancement of detoriated and superimposed pigment art : methods and case studies", In McDonald J. et Veth P., dir, A companion to rock art, Blackwell, Oxford, p. 627-643.
BREUIL H. CAPITAN L. 1902 - « Gravures paléolithiques sur les parois de la grotte des Combarelles », Bulletin de la Société d'Anthropologie de Paris, volume 3, p. 527-535.

CAPITAN L., BREUIL H. 1901 - « Une nouvelle grotte avec parois gravées à l'époque paléolithique », Compte-rendu de l'Académie des Sciences, Paris.

CAPITAN L., BREUIL H. 1902a - « Figures préhistoriques de la grotte des Combarelles (Dordogne) ", Compte-rendu des séances de l'Académie des Inscriptions et BellesLettres, 46, $\mathrm{n}^{\circ}$ 1, p. 51-56.

CAPITAN L., BREUIL H. 1902b - " La grotte des Combarelles. Les gravures sur les parois des grottes préhistoriques ", Revue de l'Ecole d'Anthropologie, 12, 1, Félix Alcan, Paris, p. 33-46, 8 fig.

CAPITAN L., BREUIL H. 1902c - « Les figures gravées à l'époque paléolithique sur les parois de la grotte des Combarelles ", Association française pour l'Avancement des Sciences, Montauban.

CAPITAN L., BREUIL H., PEYRONY D. 1924 - Les Combarelles aux Eyzies (Dordogne). Masson \& Cie Ed., Paris, Peintures et gravures murales des cavernes paléolithiques, 190 p., 127 fig.

CLEYET-MERLE J.-J. 1990 - Deux arguments décisifs, les Combarelles et Font-de-Gaume », in. Coll. Une histoire de la préhistoire en Aquitaine, Paleo, n¹, 1990, pp. 40-43.

CLEYET-MERLE J.-J., FERUGLIO V., DELLUC M. sous presse - Combarelles III - Paleo, numéro spécial Hommage à Norbert Aujoulat.

COLLECTIF (Groupe de Réflexion sur l'Art Pariétal Paléolithique), 1993, L'art pariétal paléolithique. Techniques et méthodes d'étude, Editions du CTHS, 427 p., 259 fig.

DEFRASNE C. 2014 - « Digital image enhancement for recording rupestrian engravings: applications to an alpine rockshelter ", Journal of Archeological science, vol. 50, p. 31-38.

HARMAN J. 2011 - Mode d'emploi de DStrech, disponible en ligne sur www.DStrech.com

HOLLMANN J.C., CRAUSE K. 2011 - « Digital imaging and the revelation of « hidden » rock art : Vaalekop Shelter, KwaZulu-Natal », Southern African Humanities, vol. 23, p. 55-76.

HUREL A. 2011 - L'abbé Breuil, un préhistorien dans le siècle, CNRS Edition, Paris.

LE QUELLEC, J.-L., HARMAN J., DEFRASNE C., DUQUESNOY F. 2103 - «DStrech et l'amélioration des images numériques : applications à l'archéologie des images rupestres », Les Cahiers de l'AARS, vol. 16, p. 177-198. 
LE QUELLEC J.-L., DUQUESNOY F., DEFRASNE C. 2015 (manuscrit accepté) - « Digital image enhancement with DSTrech (C): is complexity always necessary for efficiency ? ", Digital applications in Archeology and cultural heritage, revue en ligne (Elsevier.com).

MAN-ESTIER E. 2009 - Les Ursidés au naturel et au figuré pendant la Préhistoire. Thèse de Doctorat de Préhistoire du Museum national d'Histoire naturelle, sous la direction de Denis vialou, 1 vol., 801 p.

REIMER, P. J., BARD, E., BAYLISS, A., BECK, J. W., BLACKWELL, P. G., BRONK RAMSEY, C., GROOTES, P. M., GUILDERSON, T. P., HAFLIDASON, H., HAJDAS, I., HATTŽ, C., HEATON, T. J., HOFFMANN, D. L., HOGG, A. G., HUGHEN, K. A., KAISER, K. F., KROMER, B., MANNING, S. W., NIU, M., REIMER, R. W., RICHARDS, D. A., SCOTT, E. M., SOUTHON, J. R., STAFF, R. A., TURNEY, C. S. M., \& VAN DER PLICHT J. 2013 "IntCal13 and Marine13 Radiocarbon Age Calibration Curves 0-50,000 Years cal BP», Radiocarbon, 55, vol. 4.
RIGAUD J.P. 1984 - « Grottes des Combarelles et de Fontde-Gaume ", Gallia Préhistoire, Informations archéologiques : circonscription d'Aquitaine, t. 27, fasc. 2, p. 275.

RIVIÈRE E. Combarelles », Association française pour l'avancement des Sciences, Caen.

ROUSSOT A. 1976 - «Grotte des Combarelles. Abri du Cap Blanc ", Livret-Guide de l'excursion A4, Sud-Ouest (Aquitaine et Charente), IXe Congrès UISPP, p. 74-77, 3 fig.

VIALOU D.(dir.) 2004 - La Préhistoire, Histoire et dictionnaire, Bouquins, Editions Robert Laffont, Paris, $1631 \mathrm{p}$. 